\title{
A cautionary note on the use of chromosome conformation capture in plants
}

\author{
Suraj Jamge ${ }^{1}$, Maike Stam ${ }^{3}$, Gerco C. Angenent ${ }^{1,2}$ and Richard G. H. Immink $k^{1,2^{*}}$
}

\begin{abstract}
Background: The chromosome conformation capture (3C) technique is a method to study chromatin interactions at specific genomic loci. Initially established for yeast the 3 C technique has been adapted to plants in recent years in order to study chromatin interactions and their role in transcriptional gene regulation. As the plant scientific community continues to implement this technology, a discussion on critical controls, validations steps and interpretation of $3 C$ data is essential to fully benefit from $3 C$ in plants.

Results: Here we assess the reliability and robustness of the $3 \mathrm{C}$ technique for the detection of chromatin interactions in Arabidopsis. As a case study, we applied this methodology to the genomic locus of a floral integrator gene SUPPRESSOR OF OVEREXPRESSION OF CONSTANS1 (SOC1), and demonstrate the need of several controls and standard validation steps to allow a meaningful interpretation of $3 \mathrm{C}$ data. The intricacies of this promising but challenging technique are discussed in depth.

Conclusions: The $3 \mathrm{C}$ technique offers an interesting opportunity to study chromatin interactions at a resolution infeasible by microscopy. However, for interpretation of $3 \mathrm{C}$ interaction data and identification of true interactions, $3 \mathrm{C}$ technology demands a stringent experimental setup and extreme caution.
\end{abstract}

Keywords: 3C, Arabidopsis, Plants, Chromatin interactions

\section{Background}

Perception and response to internal and external stimuli is the fundamental nature of cellular life. The transcriptional regulatory system plays an integral role in fulfilling the needs of the cell and organism by ensuring proper gene activity. In comparison to bacteria, transcriptional control of an eukaryotic cell is far more complex, involving several layers of regulation inside the nucleus. It takes more than just the action and sufficient quantity of activator or repressor proteins to modulate gene expression.

Many modules, such as transcription factors (TFs), RNA polymerase, chromatin remodellers and associated proteins, and regulatory DNA sequences, are determinants of eukaryotic transcription [1, 2]. All together these factors create an open chromatin structure, which is essential to initiate eukaryotic gene transcription.

\footnotetext{
*Correspondence: richard.immink@wur.nl

1 Laboratory of Molecular Biology, Wageningen University \& Research,

Droevendaalsesteeg 1, 6708 PB Wageningen, The Netherlands

Full list of author information is available at the end of the article
}

General TFs recognize and bind to discrete DNA sequences (also referred to as cis-elements) located in the core promoter region close to the transcription start site (TSS). For instance, the TATA box is one such evolutionarily conserved core promoter cis-acting element found upstream of most eukaryotic genes [3-5]. Upon association of the general TFs to cis-elements, they interact with other proteins and form complexes to recruit RNA polymerase II, thereby initiating transcription. Examples of these other proteins include specific TFs, which can bind to cis-elements more distantly located from the core promoter elements [6, 7]. When these distant ciselements are involved in the specific activation of gene expression they are called transcriptional enhancers. These enhancers can be found upstream, downstream, or within introns of coding regions and are reported to be located as far as several hundred kilo bases $(\mathrm{kb})$ from the TSS $[8,9]$. These distant enhancers can come into close proximity of their target sequences by protein-mediated chromatin interaction. In this respect, transcriptional 
gene regulation relies to a great extent on proteins that bind to DNA, not only close to the genes that they regulate, but also at distal DNA sites that can interact with the transcription initiation site by looping the intervening DNA. Thus DNA looping is speculated to be crucial to allow multiple proteins to regulate the core transcriptional machinery, resulting in a correct and controlled transcriptional response [10-12].

Over the last two decades much attention has been paid to the role of chromatin and its conformation in the regulation of gene expression. Various processes, including the differential deposition of histone variants, histone modifications such as methylation and acetylation, DNA methylation, and the activity of other non-histone architectural proteins are known to regulate the structure of chromatin [13-15]. Empirical evidence add to the notion that the dynamics of higher-order chromatin conformation plays a crucial role not just in transcription, but also in other nuclear processes inherent to DNA (DNA replication, DNA repair, chromosome transmission etc.). Therefore understanding the conformation of the chromatin within the cell nucleus has become a fundamental topic in biology.

Over the years, different imaging methods have been deployed to study chromosome conformation [16, 17]. However, detailed and local analysis of chromatin contacts with these methods has been complicated due to technical constraints. For instance, scanning electron microscopy (SEM) provides high resolution, but this technology is laborious, and most importantly, not suitable to study specific loci. Light microscopy has a limited resolution $(200 \mathrm{~nm})$, and therefore is inadequate to define local chromosome conformation. Direct in vitro evidence of DNA looping has been shown using veryhigh resolution three-dimensional atomic force microscopy (AFM) [18]. Nevertheless, this method is labour intensive and an in vitro based approach. Artificial TFs fused with fluorescent proteins such as GFP do allow to spatially visualize and temporally track repetitive genome sequences in vivo, but the method still needs optimization in order to visualize unique individual loci and to detect chromatin interactions [19]. Fluorescence in situ hybridisation (FISH) is another alternative. However, this method involves stringent preparation treatments that can influence the chromatin organization itself and it was originally only suitable for the visualization of repetitive sequences [20]. Though, recent improvements and coupling of FISH with rolling-circle amplification of gene-specific circularizable oligonucleotides makes it possible to visualize the dynamics of individual loci [21]. Overall, microscopy studies have been crucial in defining chromosome territories and nuclear architecture at a single-cell level and new developments will probably allow to image individual chromatin contacts in vivo in the near future. Additionally, a new molecular approach has become available in recent years to study spatial organization of chromosomes at a high resolution, and this molecular tool is called Chromosome Conformation Capture (3C) [22].

In $3 \mathrm{C}$, chromatin in the intact nucleus is cross-linked by formaldehyde, followed by digestion with a restriction enzyme (RE) and intramolecular ligation [22]. The $3 \mathrm{D}$ conformation of the region or locus of interest is then studied by detecting ligation events occurring between non-neighbouring restriction sites. Possible interactions occurring between different chromosomal locations within the nucleus can be quantified as fused sequences by quantitative PCR [23]. The 3C method is cell population based and results in information about the relative frequency of interactions. The $3 \mathrm{C}$ method was initially developed for yeast by Dekker and co-workers and has been widely adapted to different model organisms shortly after. For plants, this method was also successfully applied to study chromatin conformation [24] and, since then it has become a powerful method to study gene looping in plants, as summarized below.

The first report exploring $3 \mathrm{C}$ in plants comes from a study in maize (Zea mays) that describes the role of a distant enhancer sequence at the $b 1$ locus. At the $b 1$ Locus, a hepta-repeat around $100 \mathrm{~kb}$ upstream of the transcription start site (TSS) appeared to interact with the TSS region in a tissue and epiallele-specific manner [8]. Since then a number of studies have highlighted the occurrence of chromosomal interactions in Thale Cress, Arabidopsis thaliana.

Crevillen et al. reported the presence and condition-dependent disruption of a chromatin loop at the FLOWERING LOCUS C (FLC) locus upon vernalization [25]. FLC, a potent floral repressor and a polycomb target gene, is under tight control of winter cold. Vernalization is a classical epigenetic process in which prolonged cold exposure quantitatively affects the time of flowering. A robust gene loop, due to an interaction between the $5^{\prime}$ and $3^{\prime}$ flanking sequences of the $F L C$ locus, has been reported and this interaction is independent of the level of $F L C$ transcript in different genetic backgrounds and genomic contexts. However, upon vernalization, within the first 2 weeks of cold exposure, the loop is disrupted and it has been proposed that this disruption is an early event in the transition of the $F L C$ locus to an epigenetically silent stage. Subsequently, other DNA contacts in the FLC locus increase in frequency under control of the COLDAIR and COLDWRAP long non-coding RNAs (LncRNAs), giving rise to polycomb-dependent and stable repression of FLC expression [26]. 
Another study by Liu et al. in 2013 reported the occurrence of a conformational change in chromosome looping at the TERMINAL FLOWER1 (TFL1) locus that appears to be regulating TFL1 transcription [27]. In this case, disruption of the gene loop between the TSS and $3^{\prime}$ distal region of the TFL 1 locus results in TFL 1 suppression.

Likewise, two independent studies have identified gene loops at another flowering related gene, FLOWERING LOCUS T $(F T)$, that are associated with the photoperiod-dependent flowering response [28, 29]. FT, a floral integrator, can unite signals from multiple pathways to induce flowering. The first study reports the occurrence of multiple loops between a distal enhancer element (that contains CCAAT boxes) and core cis regulatory sites located in the promoter of $F T(p F T)$ [28]. Nuclear Factor-Y (NF-Y) is known to bind CCAAT-boxes and these CCAAT-bound NF-Y complexes are hypothesised to come into close proximity with core $p F T$ sites, enabling improved recruitment and stabilized binding of CONSTANS (CO), together initiating photoperiod-dependent flowering in Arabidopsis [28]. A second study showed the folding of the FT locus into a three-dimensional structure, favouring interactions between two regulatory regions (named as Block $A$ and Block $C, \sim 5.6 \mathrm{~kb}$ apart) with another region called Block $I D$, an intermittent promoter region between Block $C$ and $A$ [29]. An introduced change in the promoter length of $F T$, i.e., an increase in the distance between Block $C$ and Block ID, by a T-DNA insertion, abolished the C-ID interaction and resulted in reduced chromatin interactions of Block $\mathrm{C}$ with Block $\mathrm{A}$. It is noteworthy to mention that the chromatin interactions identified in these two studies do not overlap.

Together, the $3 \mathrm{C}$ studies discussed above provide intriguing insights into the possible roles of chromatin interactions to regulate gene expression in plants, similar to the studies reported in yeast and other model organisms [30, 31].

3C provides an interesting opportunity to study in vivo chromatin interactions at a high-resolution and thus has become a standard method for studying chromatin contacts at single gene loci $[8,24]$. However, like every other method, $3 \mathrm{C}$ has its own shortcomings. The challenges and technical issues of this method can at times outweigh its advantages. Therefore a good experimental setup, rigorous controls, and unbiased data analysis are crucial for meaningful interpretation of $3 \mathrm{C}$ data. This is clearly evident from studies performed in other model organism (mammalians, yeast etc.), where several papers have highlighted the importance of necessary $3 \mathrm{C}$ controls and appropriate data analysis [23, 32-35]. However, cautionary notes are largely missing in the plant science community. In this study we assessed the reliability and robustness of the qPCR-based 3C method in Arabidopsis. Based on this investigation, we provide detailed guidelines on necessary controls and how interaction data should be interpreted in a $3 \mathrm{C}$ experiment. Intricacies of this promising but challenging technique are further discussed.

\section{Results and discussion}

\section{Chromosome conformation capture (3C) in Arabidopsis}

To assess the reliability and robustness of the $3 \mathrm{C}$ technique for the detection of chromatin interactions in Arabidopsis, we used this methodology to investigate the chromatin conformation at the locus of the floral integrator gene SUPPRESSOR OF OVEREXPRESSION OF CONSTANS1 (SOC1) [36]. Initially, the SOC1 locus, including the $\sim 3.8 \mathrm{~kb}$ promoter, the gene body, and $\sim 1 \mathrm{~kb}$ downstream region, was divided into fragments using the fourcutter RE FspBI, as schematically shown in Fig. 1. Twelve distinct fragments of varying lengths (smallest fragment IX of $276 \mathrm{bp}$ and longest fragment VII of $1475 \mathrm{bp}$ ), spanning the entire SOC1 locus, were tested for chromatin contacts. Fragment VII, which contains the transcriptional start site (TSS), was used as the bait (also referred to as $3 \mathrm{C}$ anchor) to generate a chromatin interaction profile (Fig. 1). Throughout this study, proper controls were used as described previously [24] (also see "Methods" section) to ensure that only valid chromatin contacts are detected and quantified. As seen in Fig. 1, multiple contacts between the anchor and other regions of the $\mathrm{SOC1}$ locus were identified. Overall the $3 \mathrm{C}$ interaction profiles observed were consistent and reproducible across independent biological samples.

In a $3 \mathrm{C}$ experiment the fragment(s) that show(s) the highest interaction frequency with the bait fragment is (are) considered as chromatin contact(s). For the TSS region in the SOC1 locus the highest interaction frequencies were observed with fragments X, XI and XII, all downstream of the VII-bait (Fig. 1). Furthermore, a potential contact with a promoter region, Fragment IV, was identified.

\section{Cross-validation of chromatin contacts}

One way to validate the putative chromatin contacts identified from a $3 \mathrm{C}$ experiment, is by performing a reciprocal $3 \mathrm{C}(\mathrm{r} 3 \mathrm{C})$ experiment. In an $\mathrm{r} 3 \mathrm{C}$ experiment, the fragment showing the highest interaction frequency with the $3 \mathrm{C}$ bait in the initial assay is used as a new 3C bait to generate an interaction profile. Thus we performed $\mathrm{r} 3 \mathrm{C}$ experiments using one of the potential interacting fragments downstream of the SOC1 TSS as bait (Fragment X; Fig. 1) aiming to verify the identified contacts. The chromatin interaction profile generated using fragment $X$ as bait is shown in Fig. 2. As expected, we 


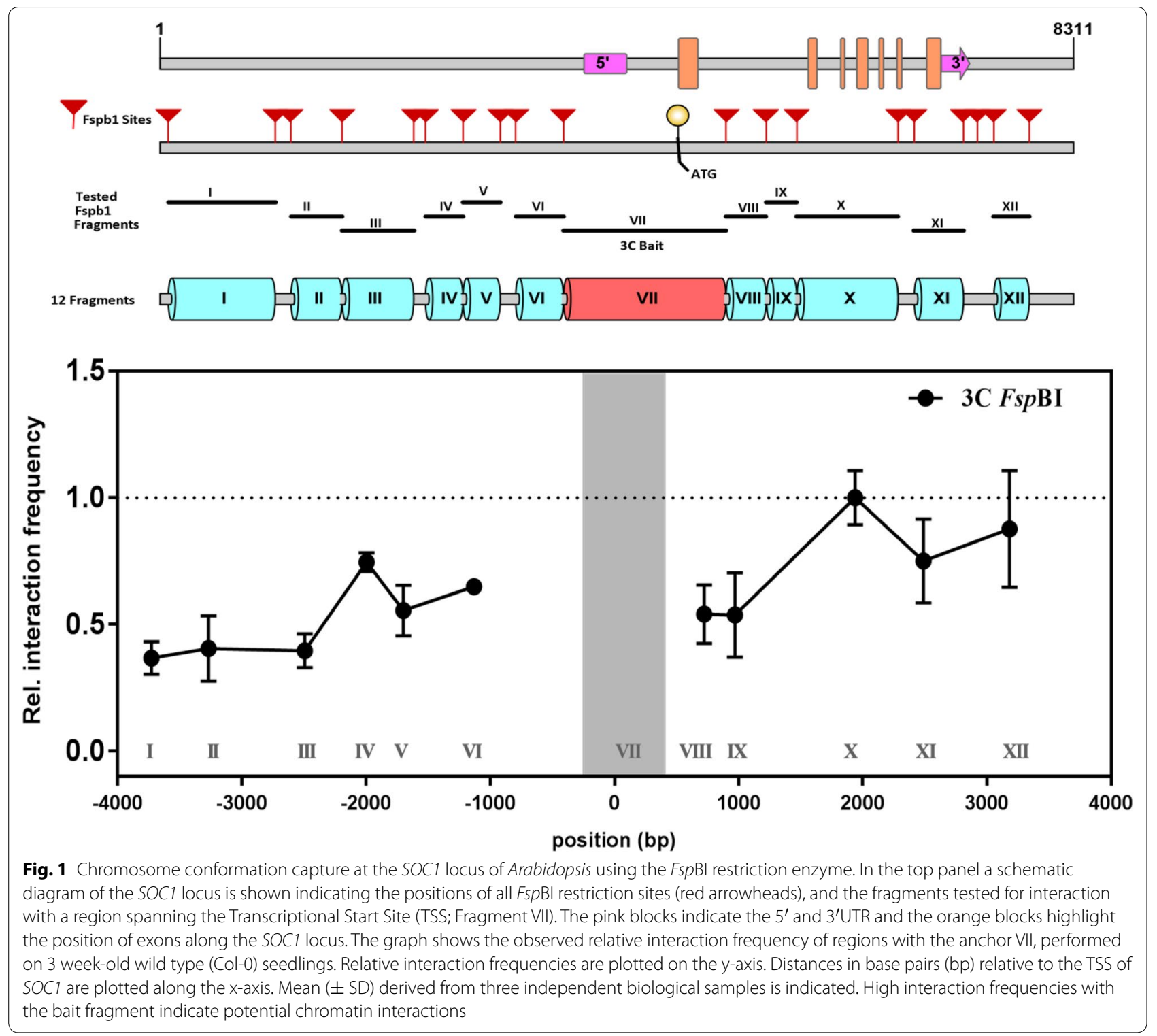

identified a high interaction frequency for the combination X-VII, validating the initial identified $3 \mathrm{C}$ contact point (Fig. 1). Moreover, another region (XI) showed an equally high interaction frequency when $\mathrm{X}$ was used as bait. However, since XI is in immediate proximity of the $3 \mathrm{C}$ bait, this high interaction frequency might be the result of random collisions of neighbouring fragments, a phenomenon often observed in 3C experiments [32].

The key difference between the $3 \mathrm{C}$ and $\mathrm{r} 3 \mathrm{C}$ experiment is the fragment that is selected as bait and consequently, the combinations of qPCR primers used to detect the relative interaction frequency. For the $S O C 1$ locus we identified in this way e.g., an interaction between bait VII and fragment $\mathrm{X}$ (Fig. 1) and therefore fragment $\mathrm{X}$ was used as bait in the r3C experiment (Fig. 2). In any PCR-based 3C experiment, the primer of the bait is kept constant and is combined with a unique primer annealing specifically to one of the fragments that is tested for interaction [8]. It is good to realize that in a $3 \mathrm{C}$ and $\mathrm{r} 3 \mathrm{C}$ experiment the combination of primers to test the interactions between one specific combination of fragments (VII and X in the example of SOC1), is identical. Performing an r3C experiment is certainly of value, since a comprehensive profile of chromatin interaction of the locus will be obtained from yet another viewpoint. However, it is good to realize that the outcome of $3 \mathrm{C}$ experiments might be biased due to different characteristics of the used REs or technical constraints of PCR [37]. Therefore, it is desirable to 


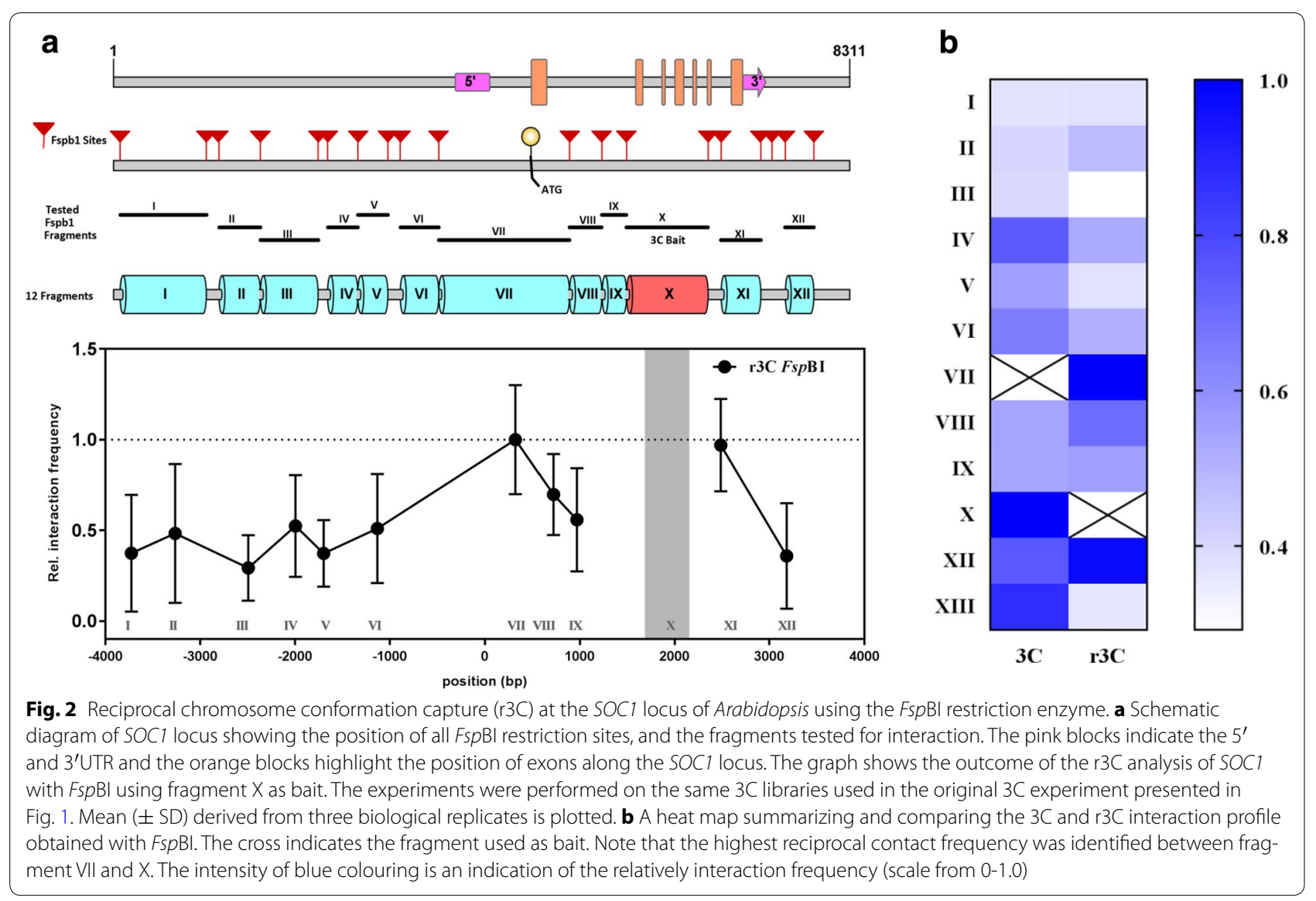

perform another independent type of validation, besides the $\mathrm{r} 3 \mathrm{C}$ experiment.

\section{Validation of $3 \mathrm{C}$ interaction profiles with another restriction enzyme}

One of the best options to confirm and validate the outcome of a $3 \mathrm{C}$ experiment is to repeat the $3 \mathrm{C}$ experiment with yet another RE. A similar $3 \mathrm{C}$ interaction profile obtained from two independent REs strengthens the reliability of identified chromatin contacts. Further, it allows a more precise identification of the specific chromosomal regions that interact. With this in mind, we re-examined the chromatin interaction profile for the SOC1 locus using a different four-cutter enzyme (NlaIII). Now 16 distinct fragments of varying length spanning the entire SOC1 locus were tested for chromatin contacts (Fig. 3). The interaction profile of this validation $3 \mathrm{C}$ assay is shown in Fig. 3a. Fragment I spanning the TSS, which to a large part overlaps with Fragment VII used as bait upon the Fsp BI digestion (Fig. 1), was used as bait. The highest interaction frequency was observed for the combination 'I-N'. In addition to that, bait I also interacted with fragments $\mathrm{L}$ and $\mathrm{C}$ at a relatively high frequency. By comparing the $3 \mathrm{C}$ profiles obtained with the two REs (Fig. $3 \mathrm{~b}$ ), we identified at least one contact to overlap in both 3C experiments with the region spanning the TSS, and this is represented by the regions $\mathrm{X}$ and L. However, region $\mathrm{N}$ was found as novel interacting region for bait $\mathrm{I}$, but this region was not represented in the $F s p B I$ run due to multiple closely located $F s p$ BI restriction sites. Consequently, this part of the locus became too fragmented for reliable qPCR primer design and amplification and was not monitored in the FspBI-based experiment. Besides this lack of coverage of some regions due to the selected RE, tested fragments do not completely overlap and this can result in differences. In the case of SOC1 for example, it is possible that the interaction between bait VII and region XII detected in the Fsp BI experiment is due to a contact between a sequence in the $5^{\prime}$-end of bait VII, and therefore not identified in the NlaIII experiment fragment I (Fig. 3b).

Although not all potential interactions were validated, the results obtained with the second RE supports the initially identified interaction between a fragment around the SOC1 TSS and a fragment towards the end of the coding region of the gene (X for $F s p \mathrm{BI}$ and $\mathrm{L}$ for NlaIII, respectively). The next logical step was to perform an $\mathrm{r} 3 \mathrm{C}$ experiment using NlaIII. For this purpose 


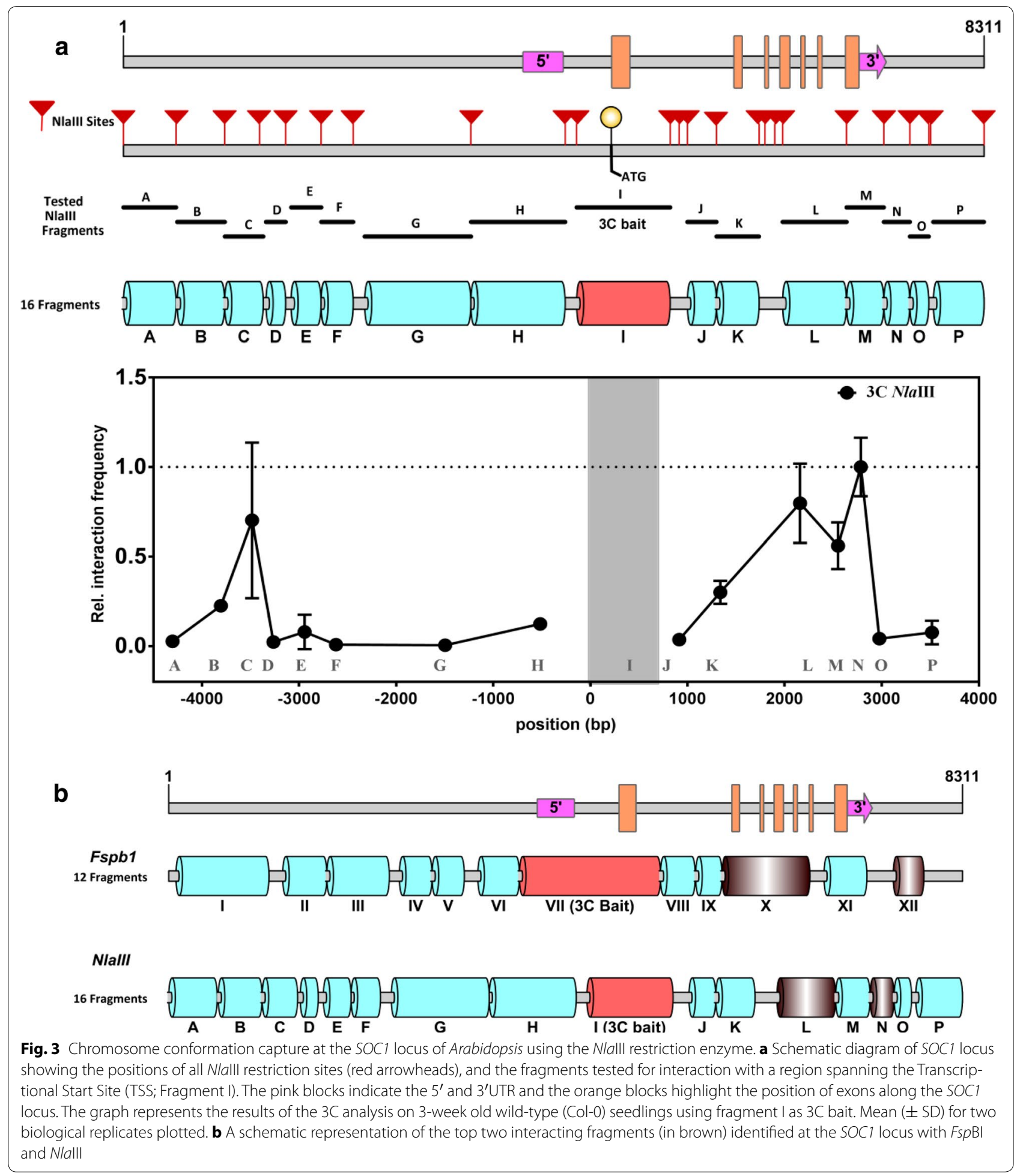

fragment N, located in the $3^{\prime}$-region of SOC1 (Figs. 3 and 4a) was used as a bait, as this region showed the highest interaction frequency with region I (Fig. 3a). Surprisingly, the observed relative interaction frequency for the combination $\mathrm{N}-\mathrm{I}$ in the $\mathrm{r} 3 \mathrm{C}$ experiments was extremely low, suggesting no interaction (Fig. 4). Instead we identified two other potential contact points, both located in the SOC1 promoter, i.e., fragments A and D. Notably, we 


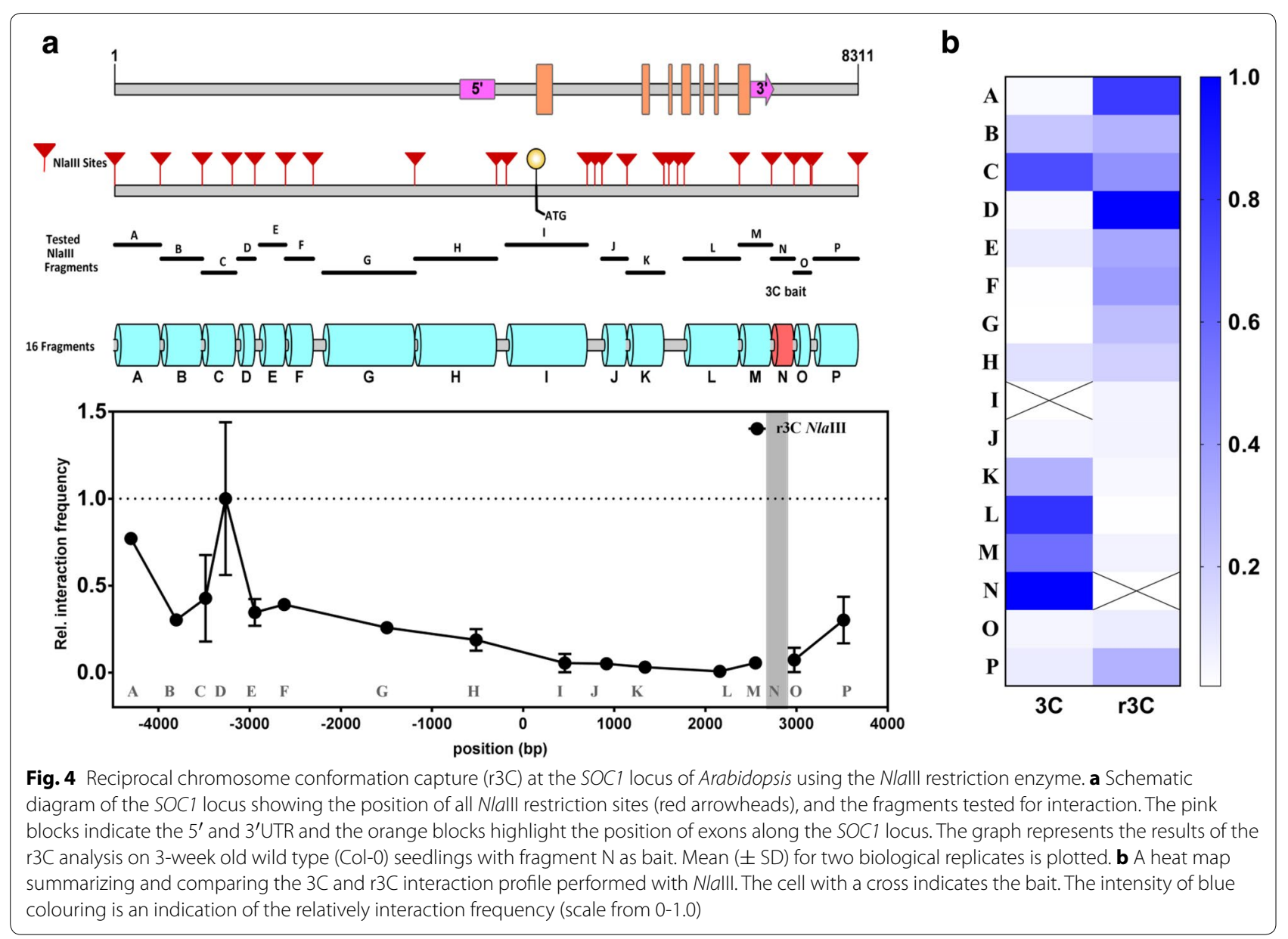

obtained a similar deviating result in all biological replica's that were tested. Although we cannot exclude the possibility that this result is due to the way interaction frequencies are calculated and interpreted, the outcome (Fig. 4) shows the importance of applying multiple validation and confirmation experiments, such as $\mathrm{r} 3 \mathrm{C}$ and the use of a second RE.

The observed contradicting results prompted us to investigate potential reasons of miss-interpretation of $3 \mathrm{C}$ results due to the lack of sufficient controls or technical constrains of the $3 \mathrm{C}$ technology. A user of $3 \mathrm{C}$ defines e.g., the bait region and the size of the region of interest to study, and hence the number of potentially interacting fragments to be monitored. These choices affect the 3C outcome, since the measured interactions are relative to one another with the highest interaction frequency set as one. In literature, we commonly come across $3 \mathrm{C}$ studies focusing on promoter regions only, the entire gene locus, or a specific distal enhancer region to identify e.g., promoter-enhancer contacts. When we re-analyzed our data starting from the hypothesis that there is an interaction between the TSS and an upstream SOC1 promoter region, and therefore monitored this part of the locus only, we observed the highest interaction between bait VII and promoter fragment IV with the $F s p B I$ restriction profile (Fig. 5). Although this chromosome interaction was also detected in our initial experiment (Fig. 1), our attention was directly drawn towards the region in the $3^{\prime}$-end of the $S O C 1$ gene, for which the highest relative interaction frequencies were found using bait VII. However, more worrying is the non-overlapping pattern at the SOC1 promoter observed for the NlaIII restriction profile, in which bait I interacts with promoter fragment C (Figs. 3a, 5). Surprisingly, this interaction pattern is not overlapping at all with the $F s p B I$ relative interaction pattern. Once more, it is possible that this deviation is caused by the lack of full overlap between the bait fragments. Sequences in the 3 '-end of fragment I might be essential for the interaction with fragment $C$. Nevertheless, these results show how much the outcome depends on the choice of the RE to be used and which chromosomal region is taken and by that, the inclusion or exclusion of particular high interacting regions. 

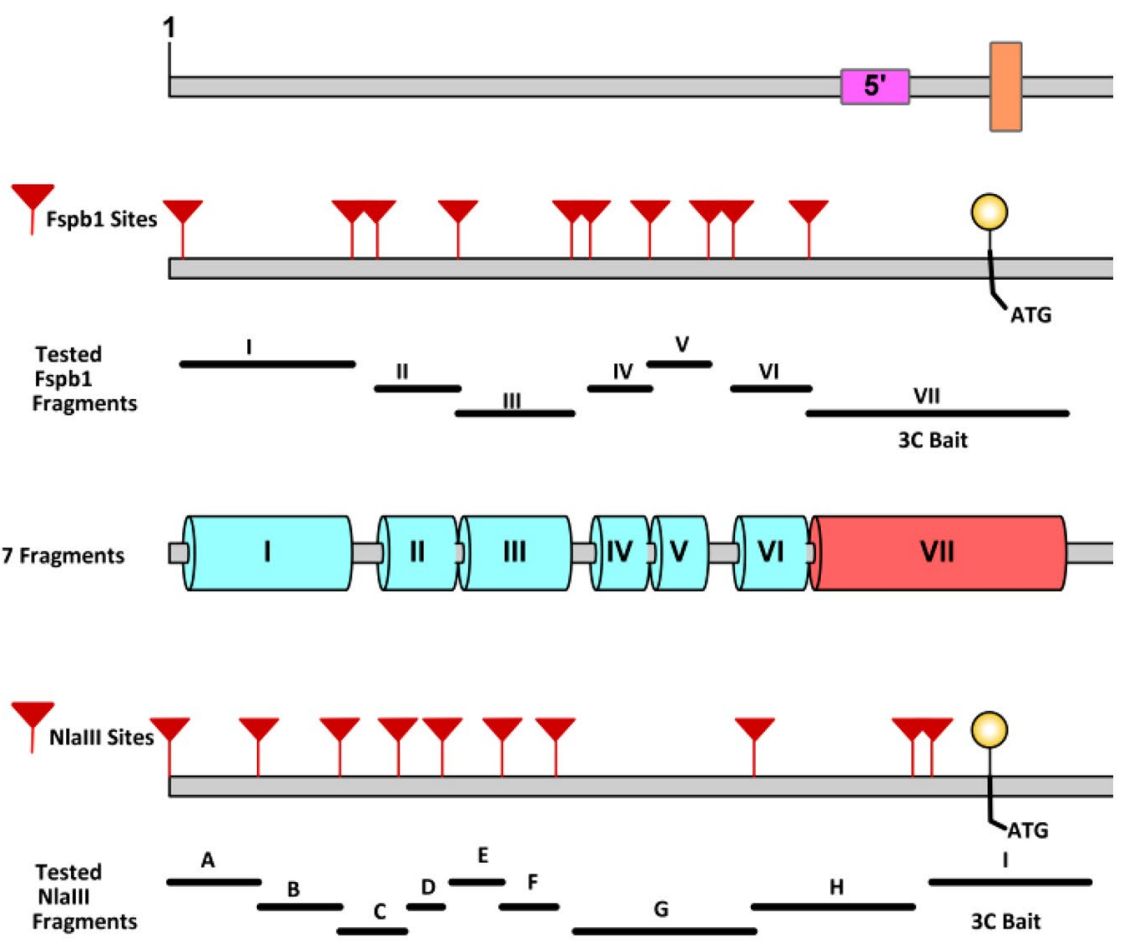

9 Fragments
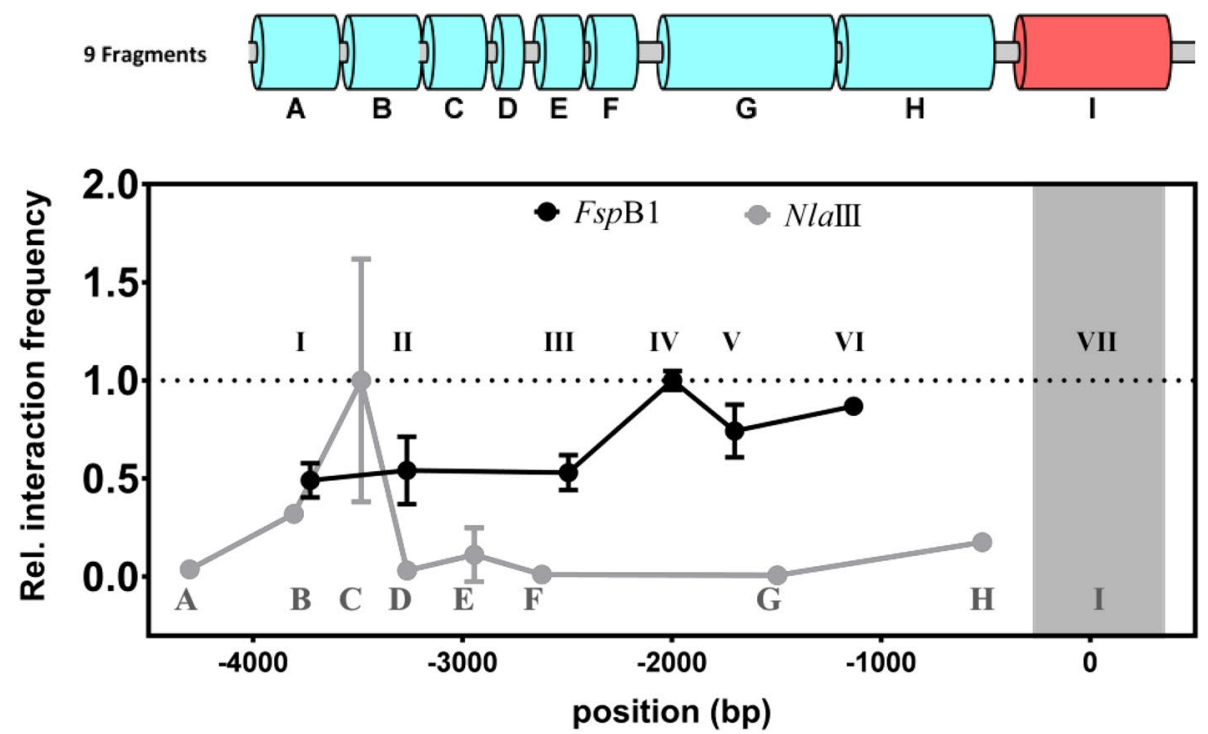

Fig. 5 Chromosome Confirmation Capture at the SOC1 promoter only. In the top panel a schematic diagram of SOC1 promoter is given, showing the position of all FspBI and N/all restriction sites, along with the fragments monitored for interaction. The graph represents the results of the $3 \mathrm{C}$ analysis based on the FspBI and N/alll data sets and using fragment VII and I as bait, respectively

Overall, upon performing $3 \mathrm{C}$ at the SOC1 locus independently with two different REs, we found various similarities, but also differences in the generated $3 \mathrm{C}$ profiles. One clear trend observed was the interaction of the TSSspanning bait region with a region close to the $3^{\prime}$ UTR of the $S O C 1$ locus for both restriction profiles. A striking discrepancy were the interaction patterns identified for the TSS bait fragment with the promoter region. Hence, our data show the potential subjectivity of $3 \mathrm{C}$ results and prompt for cautiousness when interpreting $3 \mathrm{C}$ interaction patterns. 


\section{Classification of $3 \mathrm{C}$ interactions}

All the above discussed results reveal the subjectivity of $3 \mathrm{C}$ data interpretation. Furthermore, in a $3 \mathrm{C}$ experiment, for regions in close proximity to the bait fragment under study, usually a high occurrence of ligations due to random (non-specific) collisions is observed [32, 38]. Thus, mere identification of ligation events does not necessarily mean the occurrence of an existing in vivo interaction. In order to be able to detect interactions above the background of random interactions, it's important to carefully consider the proximity between the bait and the supposed interacting fragments. The Arabidopsis genome in nature is very small and compact [39], hence studying regulatory interactions at individual gene loci is complex, due to the small distances between neighbouring restriction fragments thereby resulting in high potential for random interactions [38]. In conclusion, the combination of compact genome of Arabidopsis and the flexible nature of chromatin fibres makes interpretation of $3 \mathrm{C}$ interaction on individual loci challenging and demands high caution.

Reviewing published literature we found only a limited number of $3 \mathrm{C}$ studies that have been performed in plants. Among these studies we observed considerable shortcomings within the $3 \mathrm{C}$ experimental set up (e.g., the PCR method, lack of endogenous normalization and random ligation libraries as controls, use of only one enzyme and no $\mathrm{r} 3 \mathrm{C}$ ), and differences in the way the $3 \mathrm{C}$ interaction data have been interpreted and represented. Furthermore, comparing $3 \mathrm{C}$ results across experiments and laboratories is complicated due to differences in the experimental set up, such as PCR method, normalization method, plant growth conditions, and the way the interaction frequency is determined. For example, in literature, one can find chromatin interactions determined using $3 \mathrm{C}$ by either semi-quantitative PCR or by qPCRbased approaches. Only the latter provide numeric peak interactions and is therefore a much more trusted and widely adopted method in recent years. A recent publication manually curated more than 3000 interactions from 5000 publications across 17 species into a database called 3C database (3CDB) [40]. This 3CDB classified the strongest $3 \mathrm{C}$ interactions into four distinct classes based on their reliability. Class I and II cover the semi-quantitative PCR data, which nowadays is not an accepted detection method, whereas Class III and IV refer to numeric peak interactions. According to the $3 \mathrm{CDB}$ classification, interactions belonging to class IV are considered to be the most reliable, due to the fact that they are validated with an r3C experiment [40]. The results we described in this study are all numeric peak interactions and fall into class III and IV. However, based on our interaction profiles from independent $3 \mathrm{C}$ experiments that used different REs, we see a need to further extend this set of classifications. We suggest introducing a class $\mathrm{V}$ for interactions that have been identified and r3C-validated using two different REs. Taking into account that even in this set up the experimental procedure is the same and provides relative and hence, subjective data, $3 \mathrm{C}$ experiments should be confirmed by an alternative and independent method to get full proof for a potential chromosome interaction.

\section{Challenges of the $3 \mathrm{C}$ method}

Ligation based methods, such a $3 \mathrm{C}$, heavily rely on a sound experimental design. Many technical biases may be introduced if the design and set up of the experiment is not optimal. For example, optimization of crosslinking conditions is necessary, as over-fixed chromatin often renders digestion with REs inefficient. Similarly, biases may arises from the choice of $\mathrm{RE}$ and its digestion efficiency. One of the prerequisites for REs in $3 \mathrm{C}$ is their ability to digest crossed-linked chromatin efficiently, but at the same time providing the desired resolution at the locus of interest. By far not all REs behave optimally in $3 \mathrm{C}$, e.g., because buffering conditions during digestion are sub-optimal (e.g., due to presence of detergents). Hence, optimization of several parameters is essential in order to overcome technical biases in a PCR-based 3C experiment. Most of these technical aspects and their optimization procedures have been very-well addressed in literature [23, 24, 32, 41-43]. Besides these technical issues, biological variation may arise from differences in the growing conditions, the time of tissue collection in relation to the developmental age of the plant, and due to sampling itself. These aspects are very critical, especially when the goal is to study dynamics in chromatin interactions in time or upon a change in condition, as was e.g., done for the FLC locus [25]. To exemplify this, we performed a $3 \mathrm{C}$ assay on a developmentally different tissue where SOC1 is known to be actively expressed [44]. For this experiment we sampled the same type of plant material exactly 1 week later, but upon transfer of the plants to flowering-inducing long day (LD) conditions and after growth at these conditions for 7 days (7dai). At this time point, we observed a distinct chromatin interaction profile at SOC1 locus, when using the NlaIII RE (Additional file 1: Fig. S1), suggesting dynamics in relation to SOC1's transcriptional state. However, when $3 \mathrm{C}$ was repeated on this sample using FspBI (Additional file 1: Fig. S2), we obtained a pattern resembling the pattern after 3 weeks growth under SD conditions (Fig. 4b), suggesting lack of dynamics. This example reveals that extra caution is required when studying dynamic interactions and that it is of utmost importance to keep biological variation at minimum. 


\section{Beyond the traditional $3 \mathrm{C}$}

Since the development of the $3 \mathrm{C}$ method, many variants of this technology have been rolled out (reviewed in $[45,46]$, Table 1). These variants enabled the user among others to study chromatin interaction at a genome-wide scale. $4 \mathrm{C}$ combines the traditional $3 \mathrm{C}$ assay with microarrays (3C-on-chip) [47], and thereby a user can examine one-to-all contacts throughout the genome, instead of exploring one-to-one locus specific contacts as is done in classical 3C experiments. Advancements and development of cost-effective sequencing techniques gave birth to a wide range of sequencing variants of the $3 \mathrm{C}$ method (Table 1), improving the overall resolution of the interaction profile. Instead of using one viewpoint, some $3 \mathrm{C}$ variants, such as multiplex 3C-seq (many-to-all) and Hi-C (all-to-all), provide the opportunity to explore genome-wide interactions from multiple viewpoints simultaneously. More and more computational tools and packages are now publically available making it easier to process and analyse the vast amount of genome-wide interaction data [48-53]. Thus in comparisons to traditional $3 \mathrm{C}$, nowadays some $3 \mathrm{C}$ variants might be more attractive, robust, and cost effective to perform. Therefore, we recommend users to compare the ins-and-outs of all these methodologies taking into account their research question, before deciding on the appropriate $3 \mathrm{C}$ method of choice (reviewed in [46]). However note that the full potential of all these variants still remains to be exploited in plants. A few studies did make use of $4 \mathrm{C}$ and $\mathrm{Hi}-\mathrm{C}$ approaches to gain insight into the three-dimensional chromatin configuration of Arabidopsis genomes [54-58]. In comparison to the majority of other plant genomes, the Arabidopsis genome is densely packed with a gene density of one gene per $4.5 \mathrm{~kb}$. However, most of the chromosome conformation capture technologies are best suited to study mid-range and long-range chromatin interaction and therefore, less suitable for Arabidopsis. Nevertheless, one Hi-C study did report contact maps of

Table 1 Overview of existing and recently developed 3C-based methods

\begin{tabular}{lll}
\hline $\begin{array}{l}\text { Ligation based chromatin capture } \\
\text { method }\end{array}$ & Application & References \\
\hline qPCR-based 3C & One-to-one & {$[23,24]$} \\
3 C-seq, 4C & One-to-all & {$[59,60]$} \\
5C & Many-to-many & {$[61]$} \\
ChIA-PET & Many-to-many & {$[62]$} \\
Multiplex 3C-seq & Many-to-all & {$[63,64]$} \\
HiCap,CHi-C & Many-to-all & {$[65-67]$} \\
Capture-C & Many-to-all & {$[68]$} \\
T2C & Many-to-all & {$[69]$} \\
Hi-C, Dnase Hi-C, Micro-C, Micro-CXL, & All-to-all & {$[67,70-73]$} \\
TCC & All-to-all & {$[74]$} \\
\hline
\end{tabular}

up to $2 \mathrm{~kb}$ resolution [57]. But, when it comes to studying short-range interactions, all the above discussed variants are limited in resolution in comparison to the (q)PCRbased $3 \mathrm{C}$ methods. Further improvements in sequencing depths, choice of REs (e.g., micrococcal nuclease, four cutters) and overcoming computational barriers may drastically improve the resolution of these technologies in the near future, enabling the generation of unbiased high-resolution chromatin interaction maps.

\section{Conclusions}

$3 \mathrm{C}$ is a powerful tool when it comes to studying chromatin interactions at a gene specific locus. However, identification of valid interactions via PCR-based $3 \mathrm{C}$ demands multiple controls and validation steps. Only when the results are consistent across the proper control and validation experiments, an interaction can be considered of high confidence. Subsequently, it is of interest to unveil for every high confidence interaction whether it exists because of the regulation of gene expression, a particular nuclear or chromatin organisation, or spatial restrictions in the nucleus or the flexible nature of chromatin. Hence, identified interactions do not reveal the underlying mechanism behind its co-localization, neither do they distinguishing if it's a functional or non-functional interaction. To shed more light on the functionality of an observed interaction, genetic studies are essential. For instance, making use of T-DNA insertion lines, or targeted disruption of the DNA regions involved in the observed interactions with CRISPR-Cas9 genome editing [75], can aid further functional characterization of identified in vivo interactions.

Since the establishment of the $3 \mathrm{C}$ technique, hundreds of potential interactions have been reported supporting the potential role of chromatin interactions in transcriptional control. As the plant scientific field is gaining momentum in deciphering this new layer of transcriptional regulation of intricate gene regulatory networks, the $3 \mathrm{C}$ technique will play a prominent role in expanding our knowledge on this new fundamental topic of plant biology. Nevertheless, utmost care should be taken in assigning meaningful $3 \mathrm{C}$ interactions, as described here.

\section{Methods \\ Plant material, growth conditions and tissue collection}

Col-0 wild type plants were grown on rock-wool for 3 weeks at $20{ }^{\circ} \mathrm{C}$ under short day (SD) conditions $(8 \mathrm{~h}$ light, $16 \mathrm{~h}$ dark). Two grams of seedling material (above ground tissues) per biological sample were collected during the afternoon hour of the day. In addition, material was sampled 7 days later and after transfer and growth of the plants for a week at long day photoperiod conditions (16 h light, 8 h dark). 
Table $23 C$ primers for $F s p B I$ restriction profile

\begin{tabular}{|c|c|c|c|}
\hline Name & Gene locus and restriction enzyme & Primer on & Seq $5^{\prime}$ to $3^{\prime}$ \\
\hline PDS6848 & SOC1_FspBI & Fragment I & AGATTCTCAAACATCAGTCGGA \\
\hline PDS6849 & SOC1_FspBI & Fragment II & ACAAAAGGAGTAGGTTTCTGGA \\
\hline PDS6850 & SOC1_FspBI & Fragment III & TGAGCTTATGACTGGTAAACTC \\
\hline PDS6851 & SOC1_FspBI & Fragment IV & GTTTTGGATTTGTCTCAACCAG \\
\hline PDS7489 & SOC1_FspBI & Fragment V & TGGTCCTCCTCCCGATATAGA \\
\hline PDS6852 & SOC1_FspBI & Fragment VI & ACGAGAGAGTGTTTGTGTCC \\
\hline PDS6847 & SOC1_FspBI & Fragment VII (Bait) & GACGTTTGCTTTGAGAGGTG \\
\hline PDS6853 & SOC1_FspBI & Fragment VIII & GCTTCATTTCATGCTCATTCC \\
\hline PDS6854 & SOC1_FspBI & Fragment IX & АCTTCTTTCTCTCGAACCTACT \\
\hline PDS6855 & SOC1_FspBI & Fragment X & AGTAAGTAAGCCTCTTGTGCT \\
\hline PDS6856 & SOC1_FspBI & Fragment XI & AGCTGCTTCTCTCTTGTTGT \\
\hline PDS6857 & SOC1_FspBI & Fragment XII & AAGGGCCTACTTTGCGATAA \\
\hline PDS7307 & TIP41_Like FspBI & Bait-TSS & GTTTCGATCTCCCAGTCATG \\
\hline PDS7308 & TIP41_Like FspBI & $-500 \mathrm{bp}$ & AACTAAACCAAAGCAAATACGA \\
\hline
\end{tabular}

Table 3 3C primers for NlallI restriction profile

\begin{tabular}{|c|c|c|c|}
\hline Name & Gene locus and restriction enzyme & Primer on & Seq $5^{\prime}$ to $3^{\prime}$ \\
\hline PDS7922 & SOC1_Nlalll & Fragment A & ACCGTTGGATGAAAGAGCAT \\
\hline PDS7923 & SOC1_NIalll & Fragment B & CGCGTCTACAGAAAGTTAACCA \\
\hline PDS7924 & SOC1_NIallI & Fragment $C$ & TGACCTTACCCACATAGAAACAC \\
\hline PDS7925 & SOC1_NIalll & Fragment D & GCCAAACCAACATCACAAAA \\
\hline PDS7926 & SOC1_NIalll & Fragment $\mathrm{E}$ & GAAAACAAAAGGAGCGAAAAA \\
\hline PDS7927 & SOC1_NIalll & Fragment $\mathrm{F}$ & TTTTTCCCACCCTTATTTCTC \\
\hline PDS7928 & SOC1_NIalll & Fragment G & CATTGCCCCATTGTCTCTGT \\
\hline PDS7920 & SOC1_NIalll & Fragment $\mathrm{H}$ & ATCCTCGAAAGCTTCCTCCT \\
\hline PDS7929 & SOC1_Nlalll & Fragment I & AATCATCTGTCTCTCTCTTTCTCAA \\
\hline PDS7930 & SOC1_NIalll & Fragment J & TGAAAATGCCAGCTTTTGAT \\
\hline PDS7931 & SOC1_NIalll & Fragment K & GAGCGGTAATGAATATAACCACAA \\
\hline PDS7932 & SOC1_NIalll & Fragment L & TTGGTTATCTTCAATCATCAACCT \\
\hline PDS7933 & SOC1_Nlalll & Fragment M & TGATTCTGAACTGCTTGTGTTATG \\
\hline PDS7934 & SOC1_NIalll & Fragment N & ATCCATTGGCCAAAAATCAA \\
\hline PDS7935 & SOC1_NIallI & Fragment $\mathrm{O}$ & GAGGCTTTTAGCCCATCAAA \\
\hline PDS7936 & SOC1_NIalll & Fragment $\mathrm{P}$ & CGACGTCGCACGATTTATTA \\
\hline PDS7939 & TIP41_Like NIallI & Bait-TSS & CCGGCCTAGTTTCATTTTAGTT \\
\hline PDS7940 & TIP41_Like NIallI & -1000 & CGAGCACAAATACAAAACCG \\
\hline
\end{tabular}

\section{Chromatin conformation capture $(3 \mathrm{C})$}

$3 \mathrm{C}$ was performed on the SOC1 locus using the previously described protocol with some adaptations for Arabidopsis [24]. Two grams of Arabidopsis above-ground seedling material was crosslinked with $2 \%$ paraformaldehyde PBS buffer under vacuum for $30 \mathrm{~min}$ (mins) on ice. The crosslinking reaction was stopped by addition of ice-cold $2 \mathrm{M}$ glycine (final concentration $=0.125 \mathrm{M}$ ) under vacuum for $5 \mathrm{~min}$ on ice. The crosslinked tissue was ground and nuclei were isolated and purified using nuclei extraction (NE) buffer. Before digestion, the purified nuclei pellets were re-suspended in $1.2 \times$ restriction buffer and treated with $0.2 \%$ SDS at $65{ }^{\circ} \mathrm{C}$ for $20 \mathrm{~min}$. Later, SDS was sequestered by incubating with $2 \%$ Triton $\mathrm{X}-100$ for $30 \mathrm{~min} .3 \mathrm{C}$ analysis was performed on the SOC1 locus using two different REs (namely FspBI and NlaIII) independently. $400 \mathrm{U}$ of RE was used for overnight digestion at $37{ }^{\circ} \mathrm{C}$. Digestion was stopped by incubation at $65{ }^{\circ} \mathrm{C}$ for $20 \mathrm{~min}$. Ligation was performed using $100 \mathrm{U}$ of T4 DNA ligase, initially at $16{ }^{\circ} \mathrm{C}$ for $5 \mathrm{~h}$, followed by room temperature for $45 \mathrm{~min}$. Reverse cross-linking was done overnight with a treatment of proteinase $\mathrm{K}$ at $65^{\circ} \mathrm{C}$. After 
reversal of the crosslinks, phenol/chloroform extraction and ethanol precipitation was performed for recovery of the DNA.

\section{$3 \mathrm{C}$ primers, controls and quantification}

All the primers used in this study are listed in Tables 2 and 3. For a detailed discussion on controls we highly recommend these published studies [32, 43]. The relative interaction frequencies of one fragment to another were calculated based on quantitative PCR (qPCR) data using SYBR Green I master mix. For the analysis of the specific ligation events, two controls were used. First, in order to correct for the primer amplification efficiencies, for each primer pair the qPCR dataset was normalized with an random ligation (RL) control sample. The RL control sample was obtained by digestion of a BAC clone containing the SOC1 locus and followed by re-ligation in small volumes to obtain all the possible random ligation events. Secondly, in order to control for the quality and quantity of each $3 \mathrm{C}$ sample, the $3 \mathrm{C}$ data needed to be further normalized to $3 \mathrm{C}$ values measured for an endogenous control locus (usually a reference gene) unrelated to the SOC1 locus. The chromatin state of such a reference gene is assumed to be stable across samples. Therefore, for each $3 \mathrm{C}$ sample, $3 \mathrm{C}$ values were also obtained for the reference gene TIP41-like. The reference gene primer amplification efficiencies were also corrected with a RL control obtained by digestion and re-ligation of a BAC clone containing the TIP41-like locus. The 3C data of $S O C 1$ was normalized to the $3 \mathrm{C}$ values measured for the TIP41-like locus to obtain relative interaction frequencies. For more details on step-by-step data analysis of the qPCR-based 3C method see [76]. All figures shown in this study are the mean of two or three independent biological samples.

\section{Additional file}

Additional file 1.Fig. S1 $3 C$ analysis at the $S O C 1$ locus 7 days after the transfer of short day grown plants to long day flowering inducing conditions (7dai) on Arabidopsis rosette tissue using the Nlall restriction enzyme. Fig. $\mathbf{S 2} 3 \mathrm{C}$ analysis at the SOC1 locus 7 days after the transfer of short day grown plants to long day flowering inducing conditions (7dai) on Arabidopsis rosette tissue using the FspBI restriction enzyme.

\section{Authors' contributions}

SJ, RGHI and GCA designed the experiments. SJ performed the experiments and acquired the data. SJ and RGHI analysed the data. SJ, MS, RGHI, GCA critically discussed the outcomes of the experiments and wrote the manuscript. All authors read and approved the final manuscript.

\section{Author details}

${ }^{1}$ Laboratory of Molecular Biology, Wageningen University \& Research, Droevendaalsesteeg 1, 6708 PB Wageningen, The Netherlands. ${ }^{2}$ Wageningen Plant Research, Bioscience, Wageningen University \& Research, Droevendaalsesteeg 1, 6708 PB Wageningen, The Netherlands. ${ }^{3}$ Swammerdam Institute for Life
Sciences, Universiteit van Amsterdam, Science Park 904, 1098 XH Amsterdam, The Netherlands.

\section{Acknowledgements}

We kindly thank Iris Hovel and Blaise Weber for their advice on the methodology. We thank the PDS group members at Wageningen University \& Research and the EpiTRAITS community, for useful ideas and fruitful discussions.

\section{Competing interests}

The authors declare that they have no competing interests.

\section{Availability of data and materials}

The dataset(s) supporting the conclusions of this article are included in this article.

\section{Ethics approval and consent to participate}

Not applicable.

\section{Funding}

We gratefully acknowledge the support from the European Commission Seventh Framework-People-2012-ITN Project EpiTRAITS, GA-316965 (Epigenetic regulation of economically important plant traits) to SBJ, MES and GCA.

\section{Publisher's Note}

Springer Nature remains neutral with regard to jurisdictional claims in published maps and institutional affiliations.

Received: 24 March 2017 Accepted: 8 November 2017

Published online: 16 November 2017

\section{References}

1. Coulon A, Chow CC, Singer RH, Larson DR. Eukaryotic transcriptional dynamics: from single molecules to cell populations. Nat Rev Genet. 2013;14:572-84.

2. Urnov FD, Wolffe AP. Chromatin remodeling and transcriptional activation: the cast (in order of appearance). Oncogene. 2001;20:2991-3006.

3. Burley SK, Roeder RG. Biochemistry and structural biology of transcription factor IID (TFIID). Annu Rev Biochem. 1996;65:769-99.

4. Patikoglou GA, Kim JL, Sun L, Yang SH, Kodadek T, Burley SK. TATA element recognition by the TATA box-binding protein has been conserved throughout evolution. Genes Dev. 1999;13:3217-30.

5. Weber B, Zicola J, Oka R, Stam M. Plant enhancers: a call for discovery. Trends Plant Sci. 2016;21(11):974-87. https://doi.org/10.1016/j. tplants.2016.07.013.

6. Krivega I, Dean A. Enhancer and promoter interactions-long distance calls. Curr Opin Genet Dev. 2012;22:79-85.

7. Marsman J, Horsfield JA. Long distance relationships: enhancer-promoter communication and dynamic gene transcription. Biochim Biophys Acta. 2012;1819:1217-27.

8. Louwers M, Bader R, Haring M, van Driel R, de Laat W, Stam M. Tissue- and expression level-specific chromatin looping at maize b1 epialleles. Plant Cell. 2009;21:832-42.

9. Marand AP, Zhang T, Zhu B, Jiang J. Towards genome-wide prediction and characterization of enhancers in plants. Biochim Biophys Acta. 2017:1860(1):131-9.

10. Ptashne M. Gene regulation by proteins acting nearby and at a distance. Nature. 1986;322:697-701.

11. Schleif R. DNA looping. Science. 1988;240:127-8.

12. Matthews KS. DNA looping. Microbiol Rev. 1992;56:123-36.

13. Rosa S, Shaw P. Insights into chromatin structure and dynamics in plants. Biology (Basel). 2013;2:1378-410.

14. Jiang $D$, Berger F. Histone variants in plant transcriptional regulation. Biochim Biophys Acta. 2017;1860(1):123-30. https://doi.org/10.1016/j. bbagrm.2016.07.002.

15. Rodriguez-Granados NY, Ramirez-Prado JS, Veluchamy A, Latrasse D, Raynaud C, Crespi M, Ariel F, Benhamed M. Put your 3D glasses on: plant chromatin is on show. J Exp Bot. 2016;67:3205-21. 
16. Daban JR. Electron microscopy and atomic force microscopy studies of chromatin and metaphase chromosome structure. Micron. 2011;42:733-50.

17. Rapkin LM, Anchel DR, Li R, Bazett-Jones DP. A view of the chromatin landscape. Micron. 2012:43:150-8.

18. Puranik S, Acajjaoui S, Conn S, Costa L, Conn V, Vial A, Marcellin R, Melzer $R$, Brown $E$, Hart $D$, et al. Structural basis for the oligomerization of the MADS domain transcription factor SEPALLATA3 in Arabidopsis. Plant Cell. 2014;26:3603-15.

19. Fujimoto S, Sugano SS, Kuwata K, Osakabe K, Matsunaga S. Visualization of specific repetitive genomic sequences with fluorescent TALEs in Arabidopsis thaliana. J Exp Bot. 2016;67:6101-10.

20. Fransz P, de Jong JH, Lysak M, Castiglione MR, Schubert I. Interphase chromosomes in Arabidopsis are organized as well defined chromocenters from which euchromatin loops emanate. Proc Natl Acad Sci USA. 2002;99:14584-9.

21. Feng CM, Qiu YJ, Van Buskirk EK, Yang EJ, Chen M. Light-regulated gene repositioning in Arabidopsis. Nat Commun. 2014;5:3027.

22. Dekker J, Rippe K, Dekker M, Kleckner N. Capturing chromosome conformation. Science. 2002;295:1306-11.

23. Hagege H, Klous P, Braem C, Splinter E, Dekker J, Cathala G, de Laat W, Forne T. Quantitative analysis of chromosome conformation capture assays (3C-qPCR). Nat Protoc. 2007;2:1722-33.

24. Louwers M, Splinter E, van Driel R, de Laat W, Stam M. Studying physical chromatin interactions in plants using chromosome conformation capture (3C). Nat Protoc. 2009:4:1216-29.

25. Crevillen P, Sonmez C, Wu Z, Dean C. A gene loop containing the floral repressor FLC is disrupted in the early phase of vernalization. EMBO J. 2013:32:140-8.

26. Kim DH, Sung S. Vernalization-triggered intragenic chromatin loop formation by long noncoding RNAs. Dev Cell. 2017;40(302-312):e304.

27. Liu C, Teo ZW, Bi Y, Song S, Xi W, Yang X, Yin Z, Yu H. A conserved genetic pathway determines inflorescence architecture in Arabidopsis and rice. Dev Cell. 2013;24:612-22.

28. Cao S, Kumimoto RW, Gnesutta N, Calogero AM, Mantovani R, Holt BF 3rd. A distal CCAAT/NUCLEAR FACTOR Y complex promotes chromatin looping at the FLOWERING LOCUS T promoter and regulates the timing of flowering in Arabidopsis. Plant Cell. 2014;26:1009-17.

29. Liu L, Adrian J, Pankin A, Hu J, Dong X, von Korff M, Turck F. Induced and natural variation of promoter length modulates the photoperiodic response of FLOWERING LOCUS T. Nat Commun. 2014;5:4558.

30. Pombo A, Dillon N. Three-dimensional genome architecture: players and mechanisms. Nat Rev Mol Cell Biol. 2015;16:245-57.

31. Levine M, Cattoglio C, Tjian R. Looping back to leap forward: transcription enters a new era. Cell. 2014;157:13-25.

32. Dekker J. The three ' $C$ 's of chromosome conformation capture: controls, controls, controls. Nat Methods. 2006;3:17-21.

33. Gavrilov AA, Golov AK, Razin SV. Actual ligation frequencies in the chromosome conformation capture procedure. PLoS ONE. 2013;8:e60403.

34. Miele A, Dekker J. Mapping cis- and trans-chromatin interaction networks using chromosome conformation capture (3C). Methods Mol Biol. 2009;464:105-21.

35. O'Sullivan JM, Hendy MD, Pichugina T, Wake GC, Langowski J. The statistical-mechanics of chromosome conformation capture. Nucleus. 2013;4:390-8.

36. Lee J, Lee I. Regulation and function of SOC1, a flowering pathway integrator. J Exp Bot. 2010;61:2247-54

37. Frohler S, Dieterich C. 3PD: rapid design of optimal primers for chromosome conformation capture assays. BMC Genom. 2009;10:635.

38. Dekker J, Marti-Renom MA, Mirny LA. Exploring the three-dimensional organization of genomes: interpreting chromatin interaction data. Nat Rev Genet. 2013;14:390-403.

39. Arabidopsis Genome Initiative. Analysis of the genome sequence of the flowering plant Arabidopsis thaliana. Nature. 2000;408:796-815.

40. Yun X, Xia L, Tang B, Zhang H, Li F, Zhang Z. 3CDB: a manually curated database of chromosome conformation capture data. Database (Oxford). 2016;2016:baw044. https://doi.org/10.1093/database/baw044.

41. Naumova N, Smith EM, Zhan Y, Dekker J. Analysis of long-range chromatin interactions using chromosome conformation capture. Methods. 2012:58:192-203.
42. Hovel I, Louwers M, Stam M. 3C technologies in plants. Methods. 2012;58:204-11.

43. Splinter E, Grosveld F, de Laat W. 3C technology: analyzing the spatial organization of genomic loci in vivo. Methods Enzymol. 2004;375:493-507.

44. Immink RG, Pose D, Ferrario S, Ott F, Kaufmann K, Valentim FL, de Folter S, van der Wal F, van Dijk AD, Schmid M, Angenent GC. Characterization of SOC1's central role in flowering by the identification of its upstream and downstream regulators. Plant Physiol. 2012;160:433-49.

45. de Wit $E$, de Laat W. A decade of $3 C$ technologies: insights into nuclear organization. Genes Dev. 2012;26:11-24.

46. Denker A, de Laat W. The second decade of $3 C$ technologies: detailed insights into nuclear organization. Genes Dev. 2016;30:1357-82.

47. Simonis M, Klous P, Splinter E, Moshkin Y, Willemsen R, de Wit E, van Steensel $B$, de Laat W. Nuclear organization of active and inactive chromatin domains uncovered by chromosome conformation capture-onchip (4C). Nat Genet. 2006;38:1348-54.

48. Schmid MW, Grob S, Grossniklaus U. HiC dat: a fast and easy-to-use Hi-C data analysis tool. BMC Bioinformatics. 2015;16:277.

49. Sato T, Suyama M. ChromContact: a web tool for analyzing spatial contact of chromosomes from Hi-C data. BMC Genom. 1060;2015:16.

50. Yardimci GG, Noble WS. Software tools for visualizing Hi-C data. Genome Biol. 2017;18:26

51. Paulsen J, Sandve GK, Gundersen S, Lien TG, Trengereid K, Hovig E. HiBrowse: multi-purpose statistical analysis of genome-wide chromatin 3D organization. Bioinformatics. 2014;30:1620-2.

52. Lajoie BR, Dekker J, Kaplan N. The Hitchhiker's guide to Hi-C analysis: practical guidelines. Methods. 2015;72:65-75.

53. Ay F, Noble WS. Analysis methods for studying the $3 \mathrm{D}$ architecture of the genome. Genome Biol. 2015;16:183.

54. Moissiard G, Cokus SJ, Cary J, Feng S, Billi AC, Stroud H, Husmann D, Zhan Y, Lajoie BR, McCord RP, et al. MORC family ATPases required for heterochromatin condensation and gene silencing. Science. 2012;336:1448-51.

55. Feng S, Cokus SJ, Schubert V, Zhai J, Pellegrini M, Jacobsen SE. Genomewide $\mathrm{Hi}-\mathrm{C}$ analyses in wild-type and mutants reveal high-resolution chromatin interactions in Arabidopsis. Mol Cell. 2014;55:694-707.

56. Grob S, Schmid MW, Grossniklaus U. Hi-C analysis in Arabidopsis identifies the KNOT, a structure with similarities to the flamenco locus of Drosophila. Mol Cell. 2014;55:678-93.

57. Wang C, Liu C, Roqueiro D, Grimm D, Schwab R, Becker C, Lanz C, Weigel D. Genome-wide analysis of local chromatin packing in Arabidopsis thaliana. Genome Res. 2015;25:246-56.

58. Grob S, Schmid MW, Luedtke NW, Wicker T, Grossniklaus U. Characterization of chromosomal architecture in Arabidopsis by chromosome conformation capture. Genome Biol. 2013;14:R129.

59. Soler E, Andrieu-Soler C, de Boer E, Bryne JC, Thongjuea S, Stadhouders R, Palstra RJ, Stevens M, Kockx C, van ljcken W, et al. The genome-wide dynamics of the binding of Ldb1 complexes during erythroid differentiation. Genes Dev. 2010;24:277-89.

60. van de Werken HJ, Landan G, Holwerda SJ, Hoichman M, Klous P, Chachik R, Splinter E, Valdes-Quezada C, OzY, Bouwman BA, et al. Robust 4C-seq data analysis to screen for regulatory DNA interactions. Nat Methods. 2012:9:969-72.

61. Dostie J, Dekker J. Mapping networks of physical interactions between genomic elements using 5C technology. Nat Protoc. 2007;2:988-1002.

62. Fullwood MJ, Liu MH, Pan YF, Liu J, Xu H, Mohamed YB, Orlov YL, Velkov S, Ho A, Mei PH, et al. An oestrogen-receptor-alpha-bound human chromatin interactome. Nature. 2009;462:58-64.

63. Stadhouders R, Thongjuea S, Andrieu-Soler C, Palstra RJ, Bryne JC, van den Heuvel A, Stevens M, de Boer E, Kockx C, van der Sloot A, et al. Dynamic long-range chromatin interactions control Myb proto-oncogene transcription during erythroid development. EMBO J. 2012;31:986-99.

64. de Almeida CR, Stadhouders R, de Bruijn MJ, Bergen IM, Thongjuea S, Lenhard B, van ljcken W, Grosveld F, Galjart N, Soler E, Hendriks RW. The DNA-binding protein CTCF limits proximal Vkappa recombination and restricts kappa enhancer interactions to the immunoglobulin kappa light chain locus. Immunity. 2011;35:501-13.

65. Dryden NH, Broome LR, Dudbridge F, Johnson N, Orr N, Schoenfelder S, Nagano T, Andrews S, Wingett S, Kozarewa I, et al. Unbiased analysis of potential targets of breast cancer susceptibility loci by Capture Hi-C. Genome Res. 2014;24:1854-68. 
66. Sahlen P, Abdullayev I, Ramskold D, Matskova L, Rilakovic N, Lotstedt B, Albert TJ, Lundeberg J, Sandberg R. Genome-wide mapping of promoteranchored interactions with close to single-enhancer resolution. Genome Biol. 2015;16:156.

67. Jager R, Migliorini G, Henrion M, Kandaswamy R, Speedy HE, Heindl A, Whiffin N, Carnicer MJ, Broome L, Dryden N, et al. Capture Hi-C identifies the chromatin interactome of colorectal cancer risk loci. Nat Commun. 2015;6:6178.

68. Hughes JR, Roberts N, McGowan S, Hay D, Giannoulatou E, Lynch M, De Gobbi M, Taylor S, Gibbons R, Higgs DR. Analysis of hundreds of cis-regulatory landscapes at high resolution in a single, high-throughput experiment. Nat Genet. 2014;46:205-12.

69. Kolovos P, van de Werken HJ, Kepper N, Zuin J, Brouwer RW, Kockx CE, Wendt KS. van IJcken WF, Grosveld F, Knoch TA: Targeted Chromatin Capture (T2C): a novel high resolution high throughput method to detect genomic interactions and regulatory elements. Epigenetics Chromatin. 2014;7:10.

70. Ramani V, Cusanovich DA, Hause RJ, Ma WX, Qiu RL, Deng XX, Blau CA, Disteche CM, Noble WS, Shendure J, Duan ZJ. Mapping 3D genome architecture through in situ DNase Hi-C. Nat Protoc. 2016;11:59-76.
71. Hsieh THS, Weiner A, Lajoie B, Dekker J, Friedman N, Rando OJ. mapping nucleosome resolution chromosome folding in yeast by micro-C. Cell. 2015;162:108-19.

72. Hsieh THS, Fudenberg G, Goloborodko A, Rando OJ. Micro-C XL: assaying chromosome conformation from the nucleosome to the entire genome. Nat Methods. 2016;13:1009-11.

73. Lieberman-Aiden E, van Berkum NL, Williams L, Imakaev M, Ragoczy T, Telling A, Amit I, Lajoie BR, Sabo PJ, Dorschner MO, et al. Comprehensive mapping of long-range interactions reveals folding principles of the human genome. Science. 2009;326:289-93.

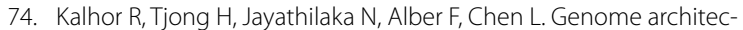
tures revealed by tethered chromosome conformation capture and population-based modeling. Nat Biotechnol. 2012;30:90-U139.

75. Woo JW, Kim J, Kwon SI, Corvalan C, Cho SW, Kim H, Kim SG, Kim ST, Choe S, Kim JS. DNA-free genome editing in plants with preassembled CRISPRCas9 ribonucleoproteins. Nat Biotechnol. 2015;33:1162-4.

76. Weber B, Jamge S, Stam M. 3C in Maize and Arabidopsis. Methods Mol Biol. 2018:1675:247-70.

\section{Submit your next manuscript to BioMed Central and we will help you at every step:}

- We accept pre-submission inquiries

- Our selector tool helps you to find the most relevant journal

- We provide round the clock customer support

- Convenient online submission

- Thorough peer review

- Inclusion in PubMed and all major indexing services

- Maximum visibility for your research

Submit your manuscript at www.biomedcentral com/submit 